\title{
DEEP OCEAN BATHYMETRIC IMAGING WITH GLORI-B
}

\author{
Russell Beale \\ School of Computer Science \\ University of Birmingham \\ Edgbaston, Birmingham \\ B15 2TT, U.K.
}

Michael Somers

\author{
Institute of Oceanographic Sciences \\ Deacon Laboratory \\ Brook Road, Wormley \\ Godalming, Surrey \\ GU8 5UB, U.K.
}

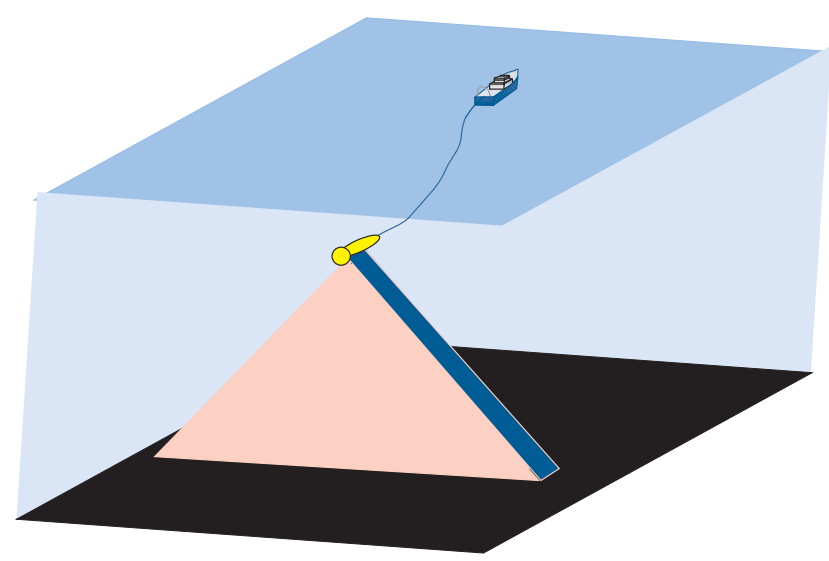

This paper describes the bathymetric imaging and processing of the GLORI-B system. GLORI-B is a deep ocean sidescan sonar surveying device, which is able to produce bathymetry measurements and images of the seafloor in swaths of up to $30 \mathrm{~km}$ wide. The basics of the GLORI-B system are described, and then the various stages of processing the received data to transform it into a geographically registered bathymetric record are given. A brief discussion of the major sources of error is also provided.

\section{INTRODUCTION}

For over twenty years the deep ocean floor has been surveyed on a large scale by the GLORIA system, developed by the Institute of Oceanographic Sciences in the UK. In that time, millions of square kilometres have been imaged, providing scientists with information about the geological process that drive the evolution of the earth's crust and allowing them to identify areas of key interest.

GLORIA (Geological LOng-Range Inclined Asdic) is a side-scan sonar device that produces images of the acoustic properties of the seafloor. Sound is used as it can penetrate to the depths of the ocean, though the anisotropic nature of the seawater medium complicates the imaging process. Recent collaboration between the UK and the U.S. Geological Survey (USGS) has resulted in an upgrade of the GLORIA system so that bathymetric data (depth measurements) can be obtained as well as the imagery. The new system is entitled GLORI-B (Geological LOng-Range Imagery and Bathymetry). This paper describes the bathymetric imaging and processing aspects of the GLORI-B system.
Figure 1: GLORIA is a towed sonar array that produces a swath of measurements perpendicular to the ship's track with every ping

\section{GLORIA SIDE-SCAN SONAR}

The GLORIA towfish contains two rows of transducers in its 2.04 ton, $7.4 \times 0.66 \mathrm{~m}$ chassis. It is neutrally buoyant, but towed at the end of a $400 \mathrm{~m}$ armoured cable it sits at approximately $50 \mathrm{~m}$ deep at a surveying speed of 8 knots. A towfish is used in order to insulate the transducers from the noise of the ship and to give greater flexibility in deployment. The transducers emit a sound pulse down into the ocean. This travels, albeit anisotropically, down to the seafloor and is reflected and scattered back. These returned echos form the basis of the data acquisition system. Young bare rock, such as recent seafloor spreading fabric, produces a high level of backscatter, whilst older rocks with a covering of sediment give lower echo returns. Areas of flat silt-covered abyssal plain act as effective absorbers and very little of the signal is returned. The strength of the signal is mapped onto an arbitrary colourmap, usually greyscale, and is used to form an image of the seafloor. 
The sound pulse used has a broad beam pattern in the vertical plane (35-40 degrees), and this produces isonification of a wide area of the seafloor. Horiontally, it is as narrow as possible -2.7 degrees. In general, signals from directly beneath the ship return first, whilst reverberations from the seabed further away from the nadir take longer to return, allowing the system to build up a picture of a wide strip of seafloor, whilst the forward motion of the ship lays down each of these lines consecutively, producing a broad swath of imagery centred along the ship's track. Typical GLORIA swaths are between 20 and $30 \mathrm{~km}$ wide.

This basic GLORIA system has been upgraded to produce the swath bathymetry, utilising over $90 \%$ of the original components. The principles are similar to those described by Hussong and Fryer[1] for the SeaMARC system in 1983. Instead of measuring just the intensity of the returned signal which is currently used to produce the imagery, the phase difference between the echo signals arriving at two closely spaced rows of receiving elements is also utilised. Knowing this phase difference allows us to deduce the angle of the returning ray, which in turn means that we can produce angle-time and hence range-depth estimates. These estimates extend out into the far- range, producing swath bathymetry up to $26 \mathrm{~km}$ wide.

\section{DATA ACQUISITION}

The upgraded system uses two rows of transducers on each side of the towfish in order to produce swath bathymetry, rather than the single row per side that is sufficient to generate the imagery. Both rows of transducers generate the sound pulse at the same time, but because they are spatially separated, the returning signal encounters one row before the other; from this the phase of the returned signal can be calculated. In order to generate sufficient power in the signal without the transducers cavitating, a $100 \mathrm{~Hz}$ linear chirp signal of 2 seconds duration is used. To produce the images, a replica correlator is used to compress the signal tails to $10 \mathrm{~ms}$. However, the effect of using a long pulse rather than a point source is that the signal is returned from upwards of $1.5 \mathrm{~km}$ of seafloor at any point in time; alternatively phrased, the phase return from one point is smeared over 2 seconds.

The solution to the phase smearing is to process in the frequency domain. The returning echo is sectioned into short timeslices and transformed by FFT to baseband (ie. centred on zero frequency). The FFT occurs separately on the two transducer rows for the port and starboard sides, and the results are stored in pairs of 2- $\mathrm{d}$ arrays of complex spectra, with frequency bin num- ber and time as indicies. The signal from a fixed single point will be a diagonal in this matrix.

A MIL-STD 1553 data transmission system is installed in the vehicle, and this instrumentation takes port and starboard upper and lower signals and produces a digital stream of baseband complex quadrature samples at $224 \mathrm{~Hz}$, oversampling at 2.24:1 to minimize alias errors and interpolation errors for the image correlators.

The 1553 sends the data up the towing cable to a standard PC card. This is then transferred to a DSP card (Texas Instruments TMS320C30 chip), which computes 64-point complex floating point radix-4 FFTs for all 4 channels every 16 samples (70ms intervals), each FFT taking $200 \mu \mathrm{s}$. Oversampling means that 28 frequency bins (of the 64) are within the sonar band. The DSP extracts the relevant ones, and with vehicle data added as a header, they are passed over ethernet using PC-NFS to a Sun for bathymetric processing.

Currently, the PC system writes hour-long files of data, which are then processed by the Unix system into bathymetric values. The processing of the bathymetry is therefore not done in real time, though this is more to do with issues of data security and developmental requirements than with the actual computational demands. It is envisaged that the subsequent versions of the software will operate in or near real time.

\section{BATHYMETRIC DATA PROCESSING}

\subsection{Phase unwrapping}

The time/frequency bin matrix containing the returned signals is skewed (i.e. time-shifted) in order to make the signal from a fixed point a vertical in the matrix. Each element of this column corresponds to one estimate of the position of the seafloor, and these estimates have to be combined to give the best possible position. Before this can occur, a number of corrections have to be applied to the values.

The transducers on the vehicle are $3 / 4$ wavelength apart, because of physical restrictions on the separation of the transducers on the towfish body. This implies that there can be some ambiguity in the received phase, since the phase angle can exceed \pm 180 degrees and is then wrapped around through zero. This ambiguity has to be resolved, and is achieved by unwrapping the phase values. In general, the first signal to be returned should be from the nadir, directly beneath the vehicle, with points further from the centerline taking correspondingly longer to return a signal: thus implies that the angle of return should be monotonic. For this system, this implies that the phase of the returned signal should be a monotonically decreasing function, 
and so from thus we can unwrap the phase values that have flipped around. The process starts from a known correct phase sample and works backwards, correcting the values that have flipped through zero. Ideally, we would like to identify a point in time at which earlier signals have wrapped phases, and later signals do not, but the presence of noise in the signal means that this point cannot be identified precisely. Problems occur if the signal is extrapolated too far back, since the early signals are water column noise. Water column noise is the term given to the background levels of noise that are ever-present in the ocean, caused by ship traffic, marine life, geological activity and so on. This noise is random and is always present in the received signal, though it is of relatively low intensity. For the first part of the return, immediately after the pulsed signal has ended, there has not been time for the sonar pulse to reach the seafloor and return, and so the only signal being received is that from the water column. It can be difficult to determine the exact onset of the first genuine return (from the nadir) from certain water column values; if the phase unwrapping is carried too far back, some values in the water column actually fit possible returned phases and so would be erroneously included as valid data points. The solution is to look at the strength of the signal as well as its phase, since the water column has a relatively low power.

The phase unwrapping is therefore complicated by two factors; the first is the presence of the water column returns. The second is noise in the return signal: the phase values fluctuate due to this noise, and so are not strictly monotonically decreasing in time; only the trend is down. We therefore used a combination of FFT curve fitting techniques with a tolerance threshold to allow for this noise, in conjunction with measurements of the signal power, calculated from the quadrature values, in order to distinguish genuine returns from water column values. The signal power and tolerance thresholds are calculated each time for each pulse, since both the early returns and far-range values are susceptible to slight variations in the parameterising of these values. Setting the power requirement too high removes much of the weak but valid far-range information, whilst if it is too low then early returns are corrupted. Setting the tolerance threshold too high removes many valid data points, whilst having it too low allows the water column to be included as a valid return. Each pulse has the water column strength analysed which is then used to determine heuristically suitable values for the tolerance and power cutoffs.

After the phase unwrapping, the time-dependant corrections can be added. The received signal has to be corrected for the movement of the towfish, of which roll is the most significant. Since the roll correction concerns the sine of arrival angle, an estimate of arrival angle is strictly necessary, but in reality for roll the period is approximately 2 seconds with an amplitude of less than 0.3 degrees so a simple correction is adequate.

We then combine all the corrected phases to form a least mean squares (LMS) estimate of the arrival angle. The LMS algorithm is useful as it only requires one arctan calculation to be performed for each separate set of return signals, and so is computationally efficient. All the signals are treated as being equally valid by using a uniform weighting, but other regimes are possible - for example, weights can be calculated according to the equality of signal amplitudes, or related to the absolute power of the received signal, but field trials suggest that these more complex calculations make little practical difference.

\subsection{Anisotropic transmission}

At this stage in the processing chain we have a set of arrival angle measurements versus time. These values have to be converted into range and depth pairings in order to produce the bathymetry. From the time taken for the signal to return to the transducers, we can determine the distance travelled, giving us the range of the reflecting surface, whilst the angle of the returning beam allows us to calculate the depth. These calculations are a variant of using a right-angled triangle, but the anisotropic nature of sound transmission in water means that the sides of the triangle are not straight lines. In order for these calculations to be accurately computed, we need to know the detailed effects of seawater on the velocity of sound. Unfortunately, this effect varies with a number of parameters; pressure, temperature, salinity and suchlike all play a role and so no one part of the ocean is identical to another. Fortunately, measurements of the velocity structure of the ocean can be carried out at intervals with relative ease, using an XBT, or eXpendable BathyThermograph. This device is basically a thermistor, with a large quantity of fine wire. Connected to a PC, it is fired off the ship and sinks down through the ocean layers returning signals from up to $600 \mathrm{~m}$ down which covers all the diurnal and seasonal variation; the regional variation below $600 \mathrm{~m}$ is generally known. From these the velocity structure can be determined, and from the velocity structure we can perform a ray-tracing exercise to produce a range estimate from any input transit time. Thus the phase-time measurements have been converted firstly to angle-time, then range-depth estimates. Note that all the stages of this processing are inherently non-linear, and are subject to alternative forms of noise and other error. 


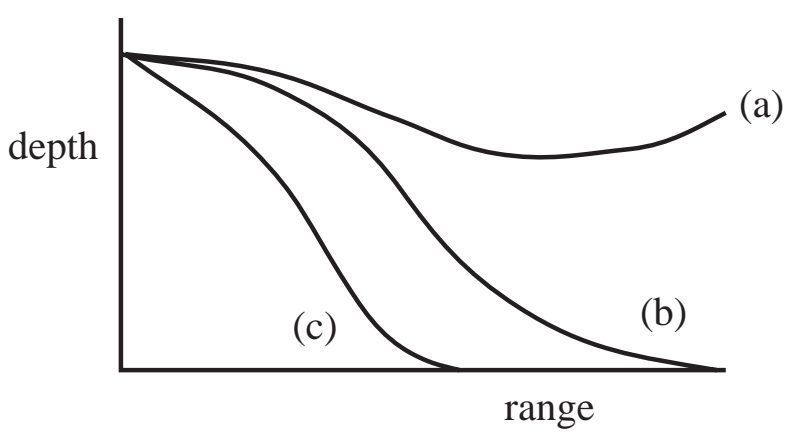

Figure 2: Sketch graph of anisotropic transmission characteristics of ocean. Ray (a) is refracted away from the seafloor, demonstrating that there is a theoretical limit on the range of isonification. Ray (b) is reflected back from the seafloor, but along a non-linear path. Ray (c) is also reflected, but suffers less from the anisotropy.

These range-depth values form the first data set, aside from the raw quadrature-sampled datastream, that we can then work with. Subsequent operations on this data set are aimed at either reducing the noise and variation exhibited by these values, or in processing the data in some other way that enhances human interpretation, for example by smoothing contours within it.

There can be many depth estimates that occur at or near a single range estimate; this is due to noise in the received phases, or by yaw in the vehicle which causes the beam to be skewed away from the perpendicular and to look slightly forwards on one side and slightly aft on the other. These estimates are processed by gridding the seafloor into cells each the size of the theoretical resolution of the GLORIA system and median filtering the results. It is also possible to filter the results in other ways, as described elsewhere, both within a single sonar ping and across pings, to remove various sources of noise or remove line dropout effects.

Each depth estimate is also coupled with the GPS (Global Positioning System) satellite data to geographically register them, assigning a latitude and longitude to every estimate.

The data can be displayed on the Sun system with a variety of bespoke software tools that present this bathymetric information in a number of ways, ranging from scatterplots of individual depths through contoured views to three-dimensional terrain maps. Onboard ship, output was primarily to a flatbed Calcomp plotter, with the option of printing onto Mylar sheets and overlaying the imagery. Post-processing onshore allows the same effects to be achieved digitally.

\section{ERRORS}

One main source of error is noise, primarily from the ship. The engine noise and propeller wash form an interference source located relatively close to the transducer array. A surface reflection (when the path of the reverberation is not towfish - seafloor - towfish but goes towfish - seafloor - sea surface - towfish) and a second bottom echo (towfish - seafloor - surface seafloor - towfish) also complicate matters. These are especially bad if it they are coherent i.e. reflected from a smooth surface, because they then introduce consistent bias rather than random noise into the readings.

Another source of error occurs due to shadowing effects. Undersea volcanos, known as seamounts, present a rapidly-changing bathymetry. However, the steep sides present an impenetrable barrier that means the far side is effectively in an acoustic shadow and so no signals are returned. However, water column noise is still present, and is detected and treated as a valid signal by the system. The beam pattern of the towfish has maximum sensitivity at approximately 20 degrees to the surface, and so the return from an area which is not isonified at all appears to be a smooth slope: increasing range sees returns at a constant angle. This is apparent on the contour record, when seamounts are clearly resolved and mapped from the ships track outwards, but their far sides all show constant, smooth slopes.

\section{CONCLUSION}

The GLORI-B bathymetric processing has been sucessfully implemented, giving full swath bathymetry capability in addition to the imagery already available from GLORIA. The received data is very noisy and subject to specific sources of error and bias, but these can be successfully modelled and hence removed from the data. The processing stages required to extract bathymetry from the raw data are modular in nature, allowing for future refinement and expansion. The computational demands are not light but the pipelined architecture suggests that a near real-time display of bathymetry could be achieved.

\section{REFERENCES}

[1] D. M. Hussong and P. Fryer. Back arc seamounts and the seamarc seafloor mapping system. EOS Trans. AGU, 64(45):627-632, 1983. 